\title{
RADIO MANIFESTATION OF THE CME OBSERVED ON APRIL 7, 2011 IN THE FREQUENCY BAND 8-32 MHZ
}

\author{
V. N. Melnik*, A. I. Brazhenko $\dagger$, G. Mann $\ddagger$ A. A. Konovalenko*, \\ A. V. Frantsuzenko ${ }^{\dagger}$, H. O. Rucker ${ }^{\S}$, and M. Panchenko
}

\begin{abstract}
On April 7, 2011 a CME was observed originating above the AR NOAA 1176, located behind the solar limb. This CME was associated with type IV burst, type II bursts and groups of J-bursts and type III bursts. Groups of J-bursts and type III bursts were observed from 10:50 to 11:20 UT. There was a group of J-bursts from 10:52 to 10:57 UT, originating from accelerated electrons propagating along high magnetic loops connected with the active region NOAA 1176 . The group of type III bursts continued from 11:00 to 11:20 UT. There were a lot of spikes and type IIIb bursts with polarizations up to $80 \%$ during the group of type III bursts. Type IV burst began at 11:20 UT at $32 \mathrm{MHz}$ and continued for more than 3 hours. Its maximum flux was about 200 s.f.u., and the polarization achieved $40 \%$. There were 3 type II bursts superimposed by type IV burst. Their drift rates were approximately $0,10 \mathrm{kHz} / \mathrm{s}, 25 \mathrm{kHz} / \mathrm{s}$, and it appears that they were radio emissions from different regions of the spherical shock produced by the CME. All type II bursts consisted of tadpoles with "heads" and "tails". Their durations were $4 \mathrm{~s}$ and $2 \mathrm{~s}$, and their polarizations were about $10 \%$ and $40 \%$, correspondingly. The frequency bands of the "tails" was up to $10 \mathrm{MHz}$. Their frequency drift rates could be positive and negative ones, and their absolute values changed from $0.4 \mathrm{MHz} / \mathrm{s}$ to $4 \mathrm{MHz} / \mathrm{s}$. Previously we found that decameter type IV bursts oscillated with periods of tens of minutes. The type IV burst observed on April 7, 2011 showed oscillations also. Fourier and wavelet analyses showed periods of about 40 minutes for both fluxes and polarizations. Moreover, it turned out that these periods decreased with time with rates of 0.03-0.07. Interpretations of all decameter phenomena are discussed.
\end{abstract}

\footnotetext{
* Institute of Radio Astronomy, Kharkov, Ukraine

$\dagger$ Poltava Gravimetric Observatory, Poltava, Ukraine

$\ddagger$ Leibniz-Institut für Astrophysik Potsdam, Solare Radiophysik, Potsdam, Germany

$\S$ Commission for Astronomy, Austrian Academy of Sciences Graz, Austria

I Space Research Institute, Austrian Academy of Sciences, Graz, Austria
} 


\section{Introduction}

Coronal mass ejections (CME) are accompanied typically by radio emission in the metric and decametric ranges [Stewart, 1985; Melnik et al., 2008]. This radio emission consists of groups of type III bursts, type II bursts and type IV burst. Sometimes, but not always, they follow in such order, but there are cases when some of these bursts are absent or superimposed on one another. This seems to connect with the power of the CME and the direction of CME propagation with regard to Earth. Because every component of radio emission has its own beaming then there are directions, which are not profitable for observation at Earth. The majority of the observed type IV bursts are accompanied by CMEs, which are governed by active regions situated at the visible solar disk. However, there are cases when type IV bursts associated with CMEs are initiated by behind-limb active regions. At the beginning of cycle 24 on April 7, 2011 we observed radio emission of these bursts associated with CMEs, which propagated behind the solar limb.

\section{Observations}

The longitude of the active region NOAA 1176 was around $140^{\circ}$, in other words it was on the back side of the Sun (Figure 1). Approximately at 10:50 UT a flare occurred and apparently initiated a coronal mass ejection (CME) (Figure 2). Its mass was $M_{C M E} \approx$ $8.3 \cdot 10^{15} \mathrm{~g}$ and the speed in the picture plane was $767 \mathrm{~km} / \mathrm{s}$ (https : cdaw.gsf c nasa.gov). Supposing that the radius of the CME core at 12:00 UT, when we observed a type IV burst (Figures 3a-3c), was about half of the solar radius, the average density in the core must be $n_{c} \approx M_{C M E} / m_{p} V_{c} \approx 2.5 \cdot 10^{7} \mathrm{~cm}^{-3}$, where $m_{p}$ is the proton mass, $V_{c}=4 \pi R_{c}^{3} / 3$ is the volume of CME core, and $R_{c}$ is the radius of the CME core.

a)

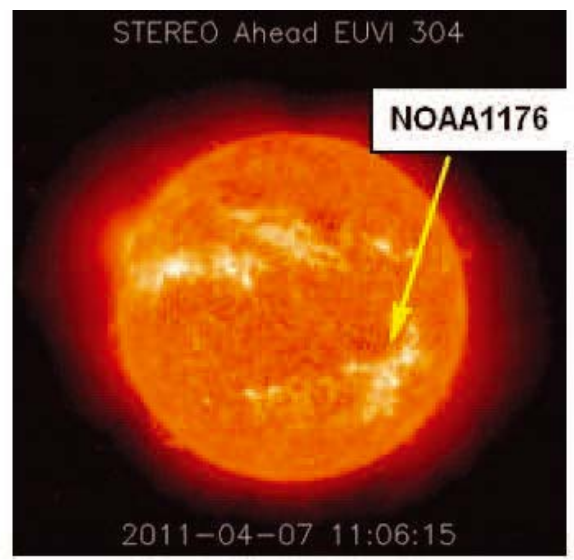

b)

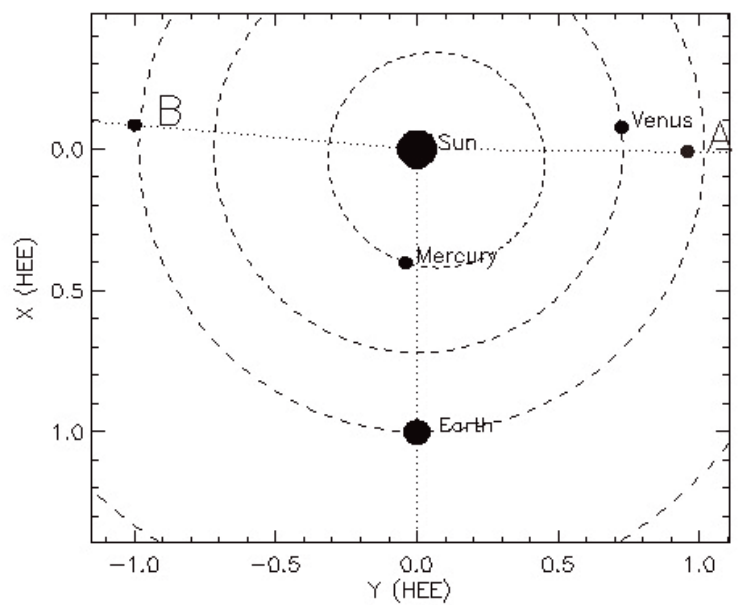

Figure 1: The active region NOAA 1176 from the viewpoint of STEREO A (a) and positions of STEREO A and STEREO B on April 7, 2011 (b).

At the same time radio emission in the form of a type IV burst was registered at the radio telescopes URAN-2 (Poltava, Ukraine) and the NDA (Nançay, France). Groups 


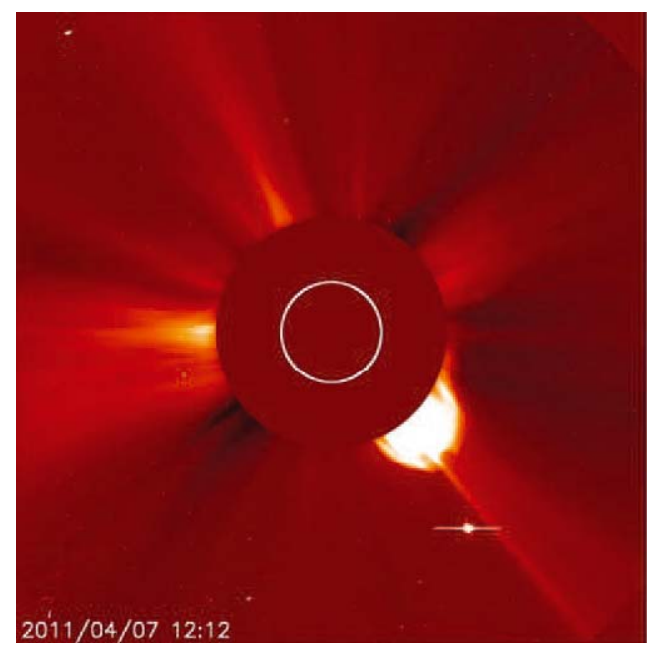

Figure 2: The coronal mass ejection originating above the AR NOAA 1176, located behind the solar limb, on April 7, 2011 at 12:12 UT.

of J-bursts [McLean, 1985; Dorovskyy et al., 2010] and type IIIb bursts [McLean, 1985] (10:52-10:57 UT) (Figure 4a), and a group of type III bursts and type IIIb bursts (11:0011:20 UT) (Figure 4b) preceded the type IV burst. Decameter spikes [Benz, 1986; Melnik et al., 2014a,b] were observed during all the time from 10:52 to 14:00 UT, especially at the time of groups of type III-IIIb bursts (Figure 4b). We separate decameter spikes as an independent type of bursts because they are situated chaotically in the dynamic spectrum [Melnik et al., 2014a,b; Shevchuk et al., 2016], in contrast to striae-bursts, which are organized into chains and form type IIIb bursts. We see that most of type III and type IIIb bursts at 11:00-11:20 were not observed at frequencies higher than 30$35 \mathrm{MHz}$ (Figure 3a), and only some of them continued up to $45 \mathrm{MHz}$. Such a feature of behind-limb bursts, so-called caterpillars, was remarked in Melnik et al. [2014a,b].

There were three successive type II bursts (burst 1, burst 2 and burst 3) from 11:22 to 11:40 UT, from 11:44 to 11:49 UT, and from 11:52 to 11:59 UT (Figure 5) with frequency drift rates equaling approximately $10 \mathrm{kHz} / \mathrm{s}, 25 \mathrm{kHz} / \mathrm{s}$, and about zero. We found frequency drift rates of type II bursts as drift rates of sequences of tadpoles (see below), because in this case such characteristic structures as backbones are absent. The burst 1 and the burst 2 were generated by shocks with linear velocities of about $300 \mathrm{~km} / \mathrm{s}$ and $800 \mathrm{~km} / \mathrm{s}$ (in the Newkirk model [Newkirk, 1961]), and the burst 3 by a shock propagating approximately parallel to the solar surface. Supposing that these shocks were initiated by the CME with a velocity of $1000 \mathrm{~km} / \mathrm{s}$ we conclude that the observed type II bursts were radio emission from flank parts of the same spherical shock (Figure 6). All type II bursts had fine structures in the form of "tadpoles" [Brazhenko et al., 2012] (Figure 7). Their properties were approximately the same for all type II bursts. The "heads" of tadpoles had durations of about $4 \mathrm{~s}$, and the polarizations were not higher than $10 \%$. The "tails" of tadpoles had durations of about $2 \mathrm{~s}$ at a fixed frequency, and their polarizations were up to $40 \%$. Some "tails" drifted to high frequencies and others drifted to low frequencies. Their drift rates changed from 0.4 to $4 \mathrm{MHz} / \mathrm{s}$. The frequency bands of "tails" were up to $10 \mathrm{MHz}$. We think that tadpoles are a manifestation of processes of electron acceleration at the shock front. Besides there is the possibility of bursty reconnection at the CME front 


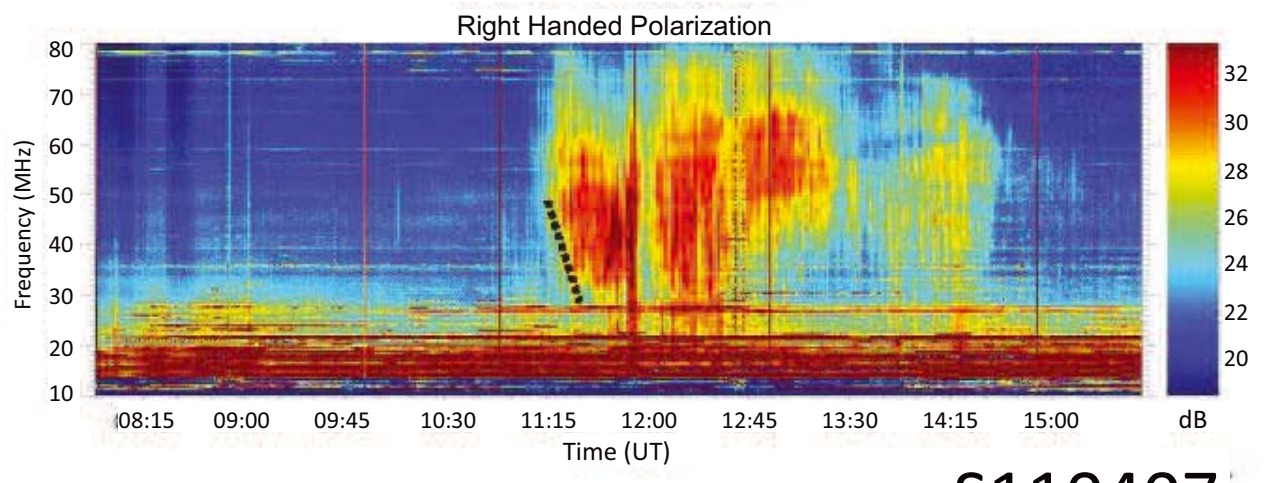

S110407

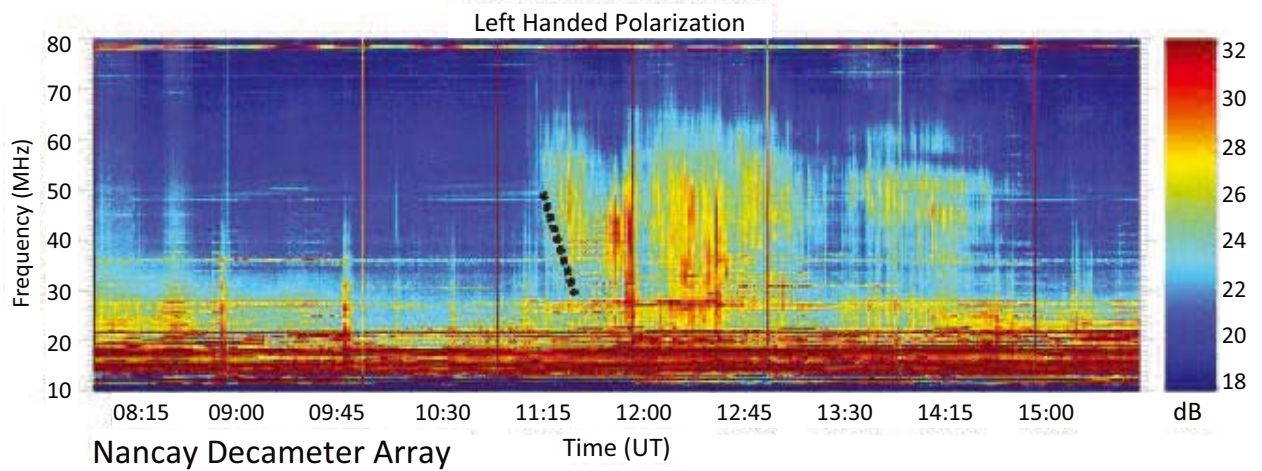

a)

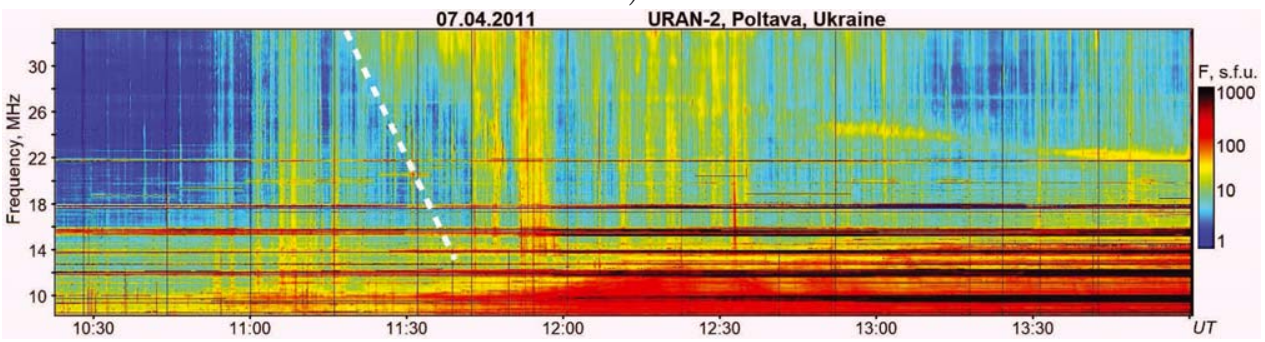

b)

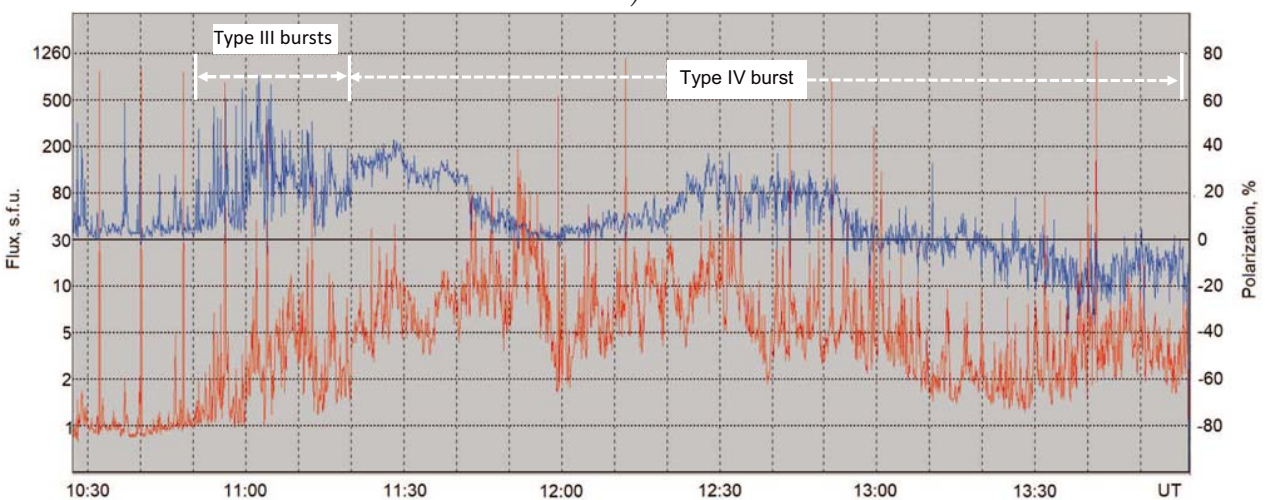

c)

Figure 3: Dynamic spectra during the CME on April 7, 2011 according to NDA (a) and URAN2 (b) radio telescopes (dotted lines show drift of forehand of type IV burst). Flux (red) and polarization (blue) profiles of group of type III bursts and type IV burst at $30 \mathrm{MHz}$ according to URAN-2 (c). 


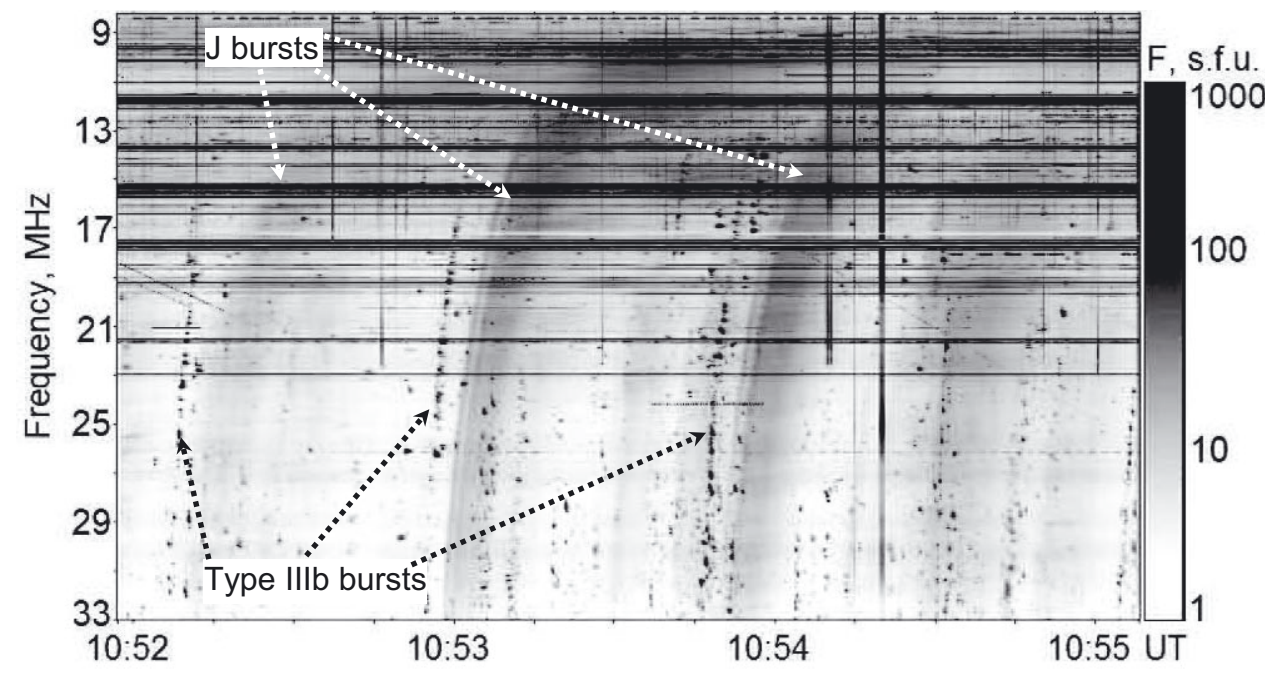

a)

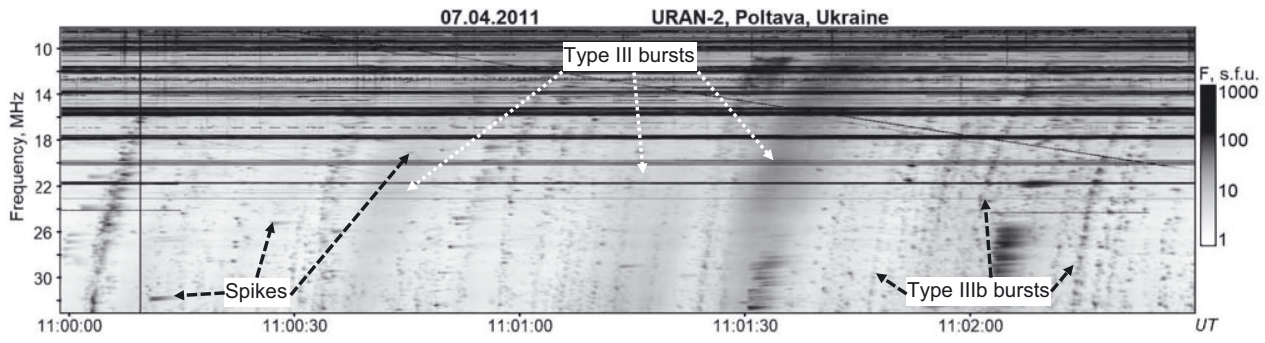

b)

Figure 4: Fragments of dynamic spectra with J-bursts, type IIIb bursts (a), and type III bursts, IIIb bursts and spikes (b).

as the CME propagates, which could produce all these bursts. We believe that there are regions similar to the Short Large Amplitude Magnetic Structures (SLAMS) observed by Mann and Luehr [1994], where electrons are accelerated. The radio emission of electrons from these regions we see as tadpole "heads". Those accelerated electrons, which cannot be confined, propagate out of these regions towards and outwards the Sun and radiate the "tails".

The type IV burst began at 11:15 UT at frequencies 40-60 MHz (Figure 3a) and at 11:20 UT at frequencies $<32 \mathrm{MHz}$ (Figure 3b). It is seen in Figure 3 that the front ridge of the type IV burst drifted with a drift rate of about $15 \mathrm{kHz} / \mathrm{s}$ at frequencies $<32 \mathrm{MHz}$, and with a drift rate of about $25 \mathrm{kHz} / \mathrm{s}$ at frequencies 30-50 MHz. The frequency band of the type IV burst extended from 12-15 MHz (Figure 3b) up to 50-70 MHz (Figure 3a). The CME core can be a source of type IV radio emission if plasma emission is the emission mechanism of this burst. As derived above, the average density of the core achieved the value $n_{c} \approx 2.5 \cdot 10^{7} \mathrm{~cm}^{-3}$. It is natural to consider that the largest density is in the center of the core and that the density decreases towards the periphery. So the density of $n_{c} \approx 6 \cdot 10^{7} \mathrm{~cm}^{-3}$ needed for radio emission at a frequency of $70 \mathrm{MHz}$ can be in the center, and the density $n_{c}<10^{7} \mathrm{~cm}^{-3}$ can be at long-distance regions from the CME core for radio emission at frequencies $<30 \mathrm{MHz}$. The frequency drift of the type IV front is determined by expansion of the core providing a negative drift rate due to the decreasing 


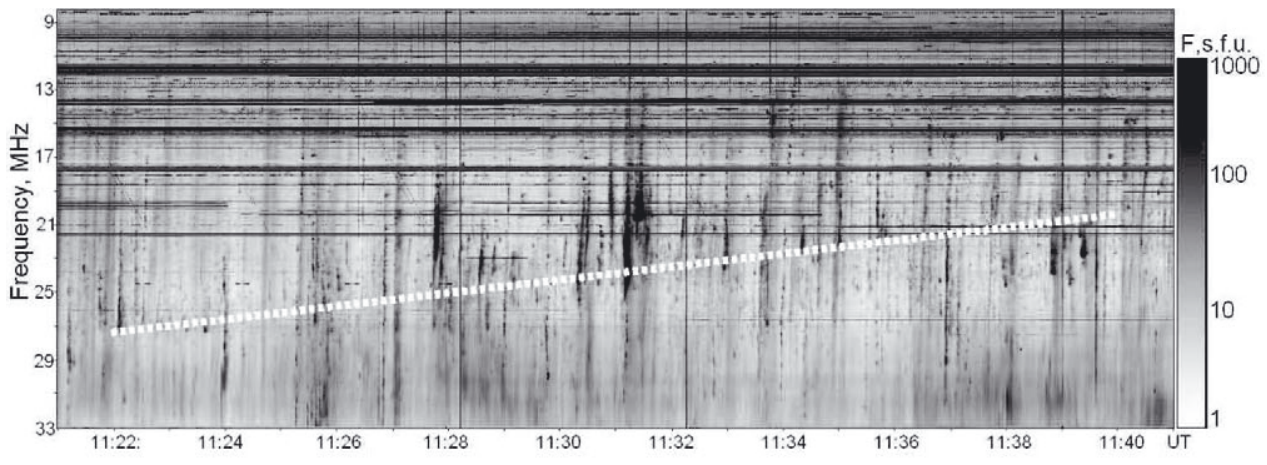

a)

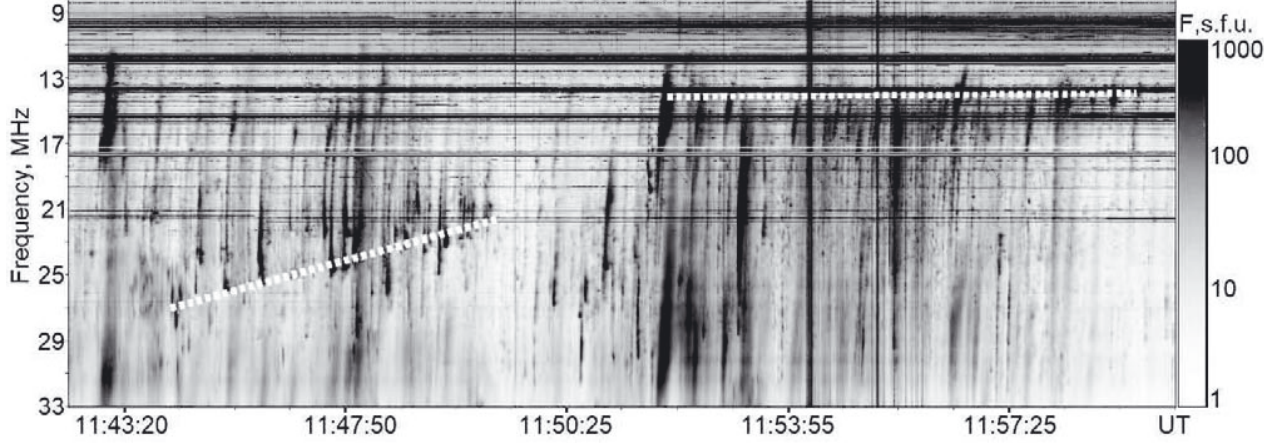

b)

Figure 5: Type II bursts consisting of "tadpoles" at 11:22-11:40 UT (the burst 1) (a), at 11:4411:49 UT (the burst 2), and at 11:51-11:59 UT (the burst 3) (b). The dotted lines show the drift of type II bursts as drift rates of tadpole sequences.

density of the periphery. The flux of the type IV burst depends on frequency and does not exceed 20-40 s.f.u., and its polarization reaches $40 \%$ (Figure 3c).

Melnik et al. [2008] reported that decameter type IV bursts oscillate with periods of tens of minutes. Zaqarashvili et al. [2013] analyzed oscillations of a decameter type IV burst which was not accompanied by a CME. Flux oscillations in that case were connected with oscillations of a high transequatorial magnetic loop. The type IV burst on April 7, 2011 was accompanied by a CME. Fourier and wavelet analyses of flux and polarization of the type IV burst showed that this burst oscillates also. In this case oscillations of the type IV burst can be governed by oscillations of the CME core. Examples of wavelet analyses at frequencies of 16.7 and $30.3 \mathrm{MHz}$ are shown in Figure 8.

The periods at the frequencies of $11.3,16.7,19.7,23.3,26.3$, and $30.3 \mathrm{MHz}$ obtained by Fourier and wavelet analyses are shown in Table 1. Oscillations in the polarization of this type IV burst are presented also (Fourier period at the frequency of $23.3 \mathrm{MHz}$ and wavelet period at the frequency of $30.3 \mathrm{MHz}$ could not be derived with confidence). We see that a period of about 40 min shows up in Fourier and wavelet analyses, both in fluxes and polarizations. At the frequency of $11.3 \mathrm{MHz}$ longer periods up to 50-60 min also appear. There is an interesting property of the period of 40 min for this type IV burst, namely that this period is decreasing with time practically linearly according to the wavelet power spectrum (Figures $8 \mathrm{a}, 8 \mathrm{~b}$ ). So the period was about $T_{1}=40 \mathrm{~min}$ at $t_{1}=12: 00 \mathrm{UT}$, and about $T_{2}=35-37$ min at $t_{1}=13: 20 \mathrm{UT}$ (high panels of Figures 8a, 8b). For the drift 


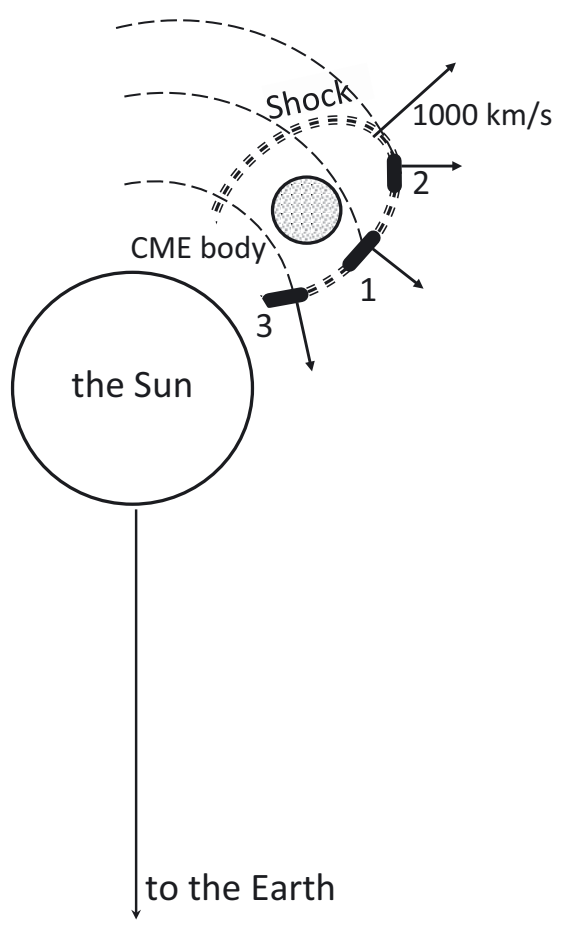

Figure 6: Sketch of CME and spherical shock initiated by this CME. Parts of the shock responsible for bursts 1, 2 and 3 are shown with directions of their propagation. The levels of corresponding plasma frequencies are shown, too.

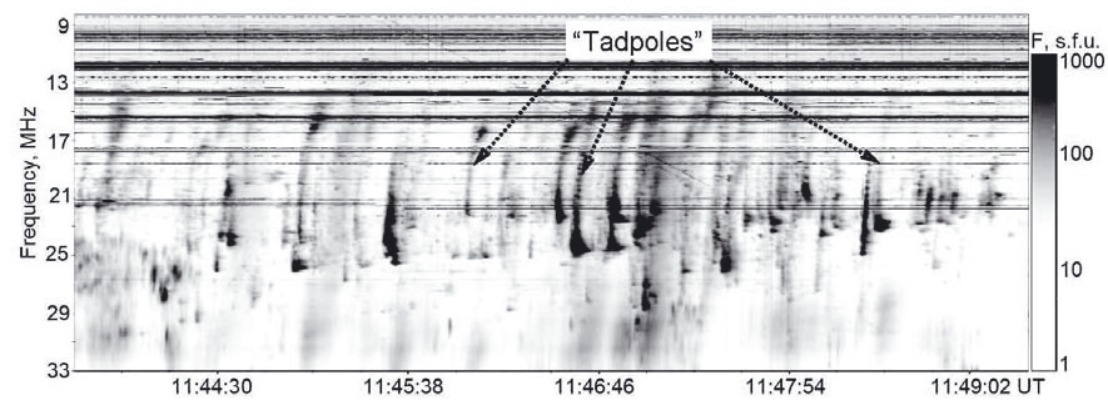

Figure 7: Tadpoles as a fine structure of type II burst.

rate of the oscillation periods $d T / d t \approx\left(T_{2}-T_{1}\right) /\left(t_{2}-t_{1}\right)$ we derived values changing from -0.03 up to -0.07 .

\section{Conclusions}

We identified decameter radio emission in the form of groups of J-bursts, type III bursts, type IIIb bursts, spikes, type II bursts, tadpoles, and type IV burst observed from 10:52 to 14:00 UT as a radio manifestation of the CME associated with the behind-limb active region NOAA 1176. This CME had a density high enough to be a source of radio emission of a type IV burst from $15 \mathrm{MHz}$ up to $70 \mathrm{MHz}$. Because the longitude of the CME was $140^{\circ}$ we conclude that the beaming of radiation of the type IV burst was wide in the meter and 

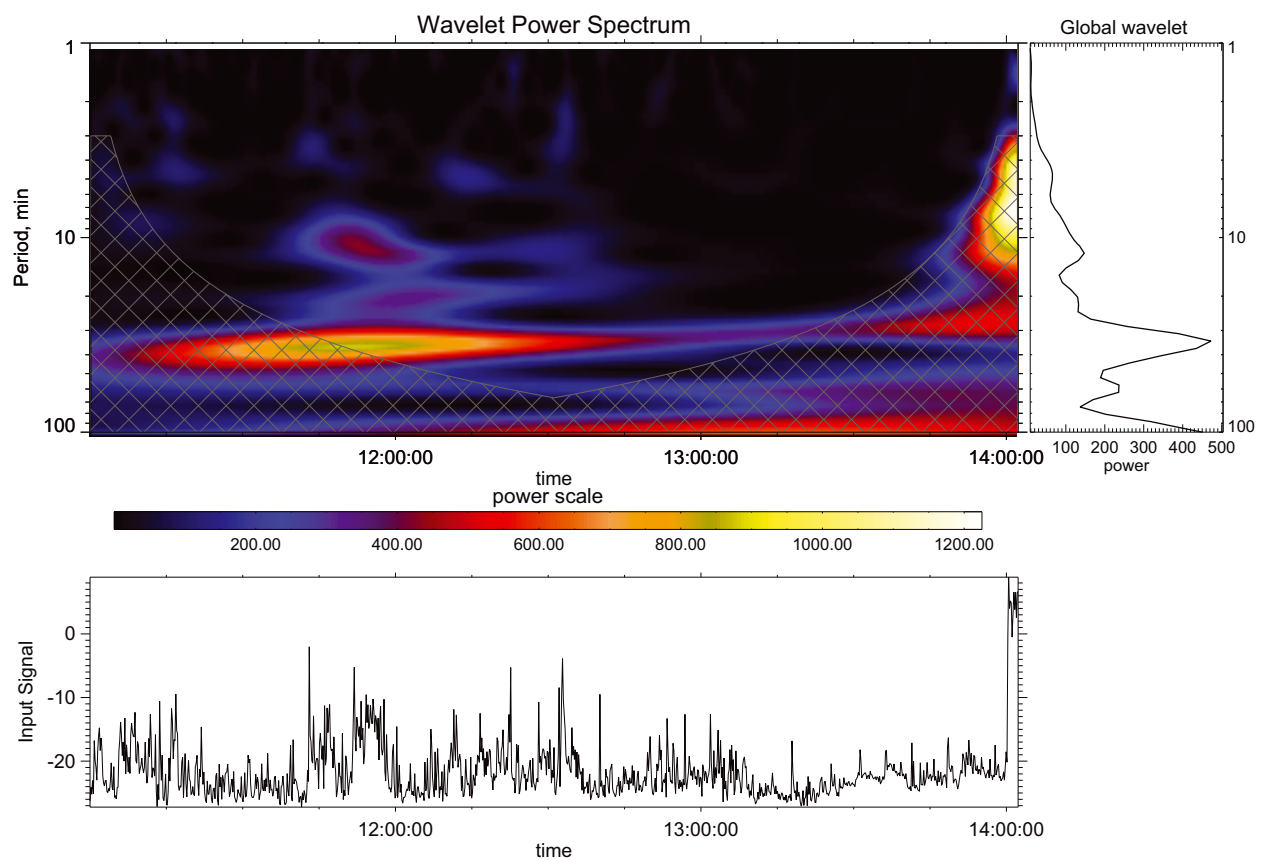

a)
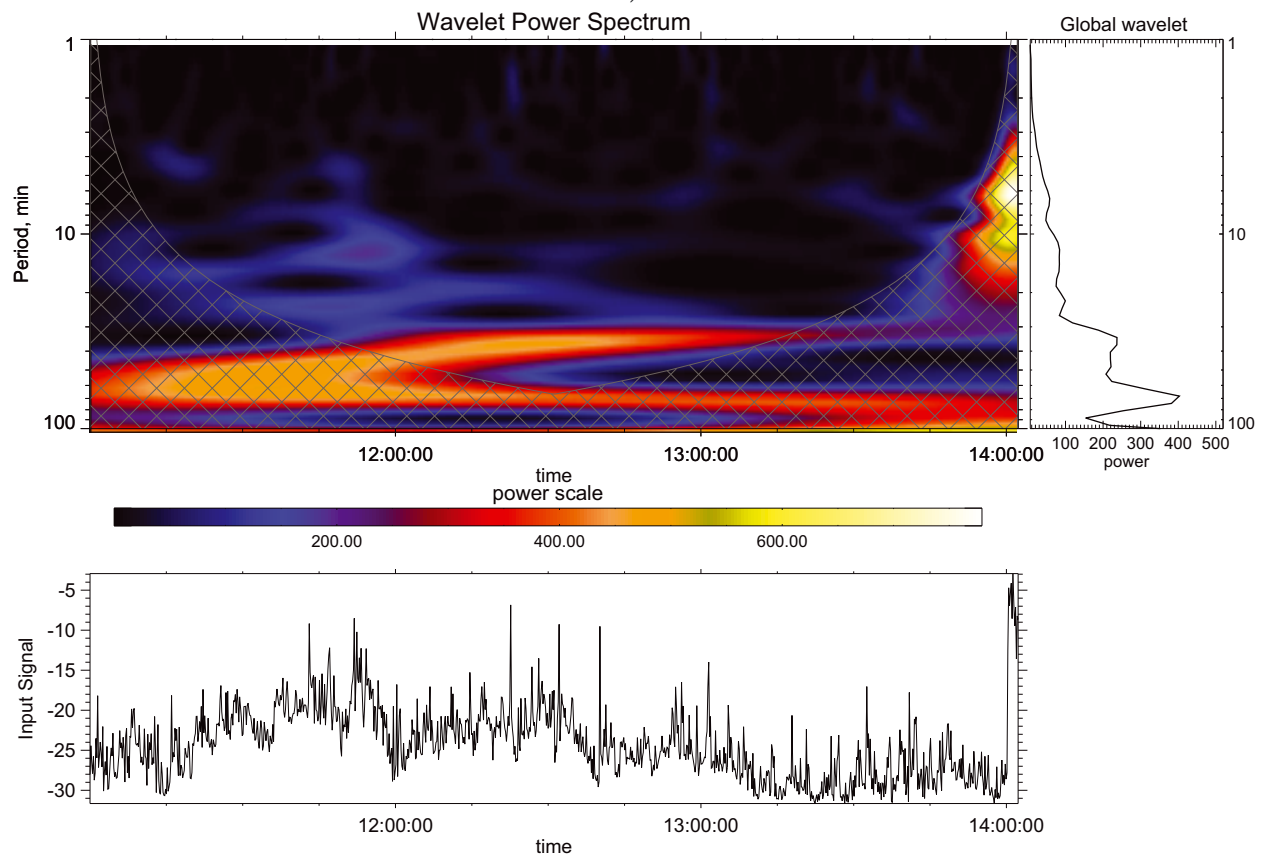

b)

Figure 8: Wavelet spectrum of radio flux at $16.75 \mathrm{MHz}$ (a) and $30.3 \mathrm{MHz}$ (b) during 10:5514:00 UT. Periods of about 40 min decreasing with time are seen at both frequencies. 
Table 1: Oscillation periods of flux and polarization of the type IV burst on April 7, 2011 at different frequencies according to Fourier and wavelet analyses.

\begin{tabular}{|c|c|c|c|c|}
\hline & \multicolumn{2}{|c|}{ Flux } & \multicolumn{2}{c|}{ Polarization } \\
\hline $\begin{array}{c}\text { Frequency } \\
(\mathrm{MHz})\end{array}$ & $\begin{array}{c}\text { Fourier period } \\
(\mathrm{min})\end{array}$ & $\begin{array}{c}\text { Wavelet period } \\
(\mathrm{min})\end{array}$ & $\begin{array}{c}\text { Fourier period } \\
(\mathrm{min})\end{array}$ & $\begin{array}{c}\text { Wavelet period } \\
(\mathrm{min})\end{array}$ \\
\hline 30.3 & 37 & $45-35$ & 33 & - \\
26.3 & 41 & $47-38$ & 45 & 46 \\
23.3 & 41 & $45-36$ & - & 44 \\
19.7 & 41,33 & $49-35$ & 41 & $40,48-43$ \\
16.7 & 41 & $35-44,46-35$ & 37 & 46 \\
11.3 & 67,33 & $45-50,41-35$ & 33 & $50-30$ \\
\hline
\end{tabular}

decameter range in contrast to that in the hectometer range [Gopalswamy et al., 2016]. The decameter type II bursts initiated by the 7 April CME seemed to be generated by flanks of a spherical shock. Fine structures in the form of tadpoles [Brazhenko et al., 2012] were characteristic for all type II bursts discussed above. "Heads" of tadpoles had durations of about $4 \mathrm{~s}$, and their "tails" had durations of about $2 \mathrm{~s}$. The "tails" drifted to high and to low frequencies. The frequency band of the "tails" was up to $10 \mathrm{MHz}$. An interpretation of these bursts can be in the fact that in some regions electron acceleration exists. In these regions electrons radiate a "head". Accelerated electrons, which can not be confined in these regions, propagating towards and outwards the Sun, generate "tails" of tadpoles. The type IV burst oscillates with a period of about $40 \mathrm{~min}$ at all frequencies from 8 to $32 \mathrm{MHz}$. Since the source of type IV radio emission can be the CME core, the oscillations of type IV burst can be a manifestation of core oscillations. If the discussed oscillations are really defined by oscillations of the CME core [Lee et al., 2015], then they can be sound or some type of magnetohydrodynamic oscillations [Nakariakov, 2004; Lee et al.,2015]. In the first case the oscillation periods are defined by the temperature of the core plasma, so we can find this temperature. In the second case, the periods are defined by the Alfvénic velocity or the magnetic field, which is present in the place of radiation, notably in the CME core. A detailed consideration of these possibilities will be discussed in a next paper.

Acknowledgments. This work was partially fulfilled in the frame of the FP7 project SOLSPANET (FP7 PEOPLE 2010 IRSES 269299). The Editors thank Atsushi Kumamoto and one anonymous reviewer for their help in evaluating this paper.

\section{References}

Benz, A. O., Millisecond radio spikes, Solar Phys., 104, 99-110, 1986.

Brazhenko, A., V. Melnik, A. Konovalenko, V. Dorovskyy, V. Vashchishin, A. Franzusenko, and H. Rucker, Observation of two coronal mass ejections on April 7, 2011 by 
radio telescope URAN-2, European Planetary Science Congress, Madrid, Spain, id.EPSC2012-544, 2012.

Dorovskyy, V.V., V. N. Melnik, A.A. Konovalenko, H.O. Rucker, E. P. Abranin, and A. Lecacheux, Solar U- and J- radio bursts at the decameter waves, in Astrophysics and Cosmology after Gamov, AIP Conference Proc., 1206, 433-439, 2010.

Gopalswamy, N., S. Akiyama, P. Mäkelä, S. Yashiro, and I. H. Cairns, On the directivity of low-frequency type IV radio bursts, preprint arXiv, 1605.02223, 2016.

Lee, H., Y.-J. Moon, and V.M. Nakariakov, Radial and azimuthal oscillations of halo coronal mass ejections in the Sun, Astrophys. J. Lett., 803, L7, 2015.

Mann, G., and H. Luehr, Electron acceleration at quasi-parallel shocks in the solar corona and its signature in solar type II radio bursts, Astrophys. J. Supplement Series, 90, 577-581, 1994.

McLean, D. J., Solar radiophysics: Studies of emission from the sun at metre wavelengths, Cambridge University Press, Cambridge and New York, 37-52, 1985.

Melnik, V. N., H. O. Rucker, A. A. Konovalenko, V. V. Dorovskyy, E. P. Abranin, A. I. Brazhenko, B. Thide, and A. A. Stanislavskyy, Solar type IV bursts at frequencies 10$30 \mathrm{MHz}$, in Solar Physics Research Trends, edited by Pingzhi Wang, Nova Science Publishers, New York, USA, 287-325, 2008.

Melnik, V.N., A. I. Brazhenko, A.A. Konovalenko, H. O. Rucker, A. V. Frantsuzenko, V. V. Dorovskyy, M. Panchenko, and A. A. Stanislavskyy, Unusual solar radio burst observed at decameter wavelengths, Solar Phys., 289, 263-278, 2014a.

Melnik, V. N., N. V. Shevchuk, A. A. Konovalenko, H. O. Rucker, V. V. Dorovskyy, S. Poedts, and A. Lecacheux, Solar decameter spikes, Solar Phys., 289, 1701-1714, 2014b.

Nakariakov, V. M., Theoretical aspects of MHD coronal seismology, in Proc. SOHO 13 - Waves, Oscillations and small-scale transient events in the solar atmosphere: A joint view from SOHO and TRACE, compiled by H. Lacoste, ESA SP-547, ESA Publ. Division, Noordwijk, Netherlands, p.407, 2004.

Newkirk, G. Jr., The solar corona in active regions and the thermal origin of the slowly varying component of solar radio radiation, Astrophys. J., 133, 983-1013, 1961.

Shevchuk, N. V., V. N. Melnik, S. Poedts, V. V. Dorovskyy, J. Magdalenic, A. A. Konovalenko, A. I. Brazhenko, C. Briand, A. V. Frantsuzenko, H. O. Rucker, and P. Zarka, The storm of decameter spikes during the event of 14 June 2012, Solar Phys., 291, 211-228, 2016.

Stewart, R. T., Moving type IV bursts, in Solar Radio Physics, edited by D. J. McLean, and N. R. Labrum, Cambridge University Press, Cambridge, UK, 361-384, 1985.

Zaqarashvili, T. V., V. N. Melnik, A.I. Brazhenko, M. Panchenko, A. A. Konovalenko, A. V. Frantzusenko, V. V. Dorovskyy, and H. O. Rucker, Radio seismology of the outer solar corona, Astron. Astrophys., 555, A55, 2013. 